\title{
Coming together
}

\author{
Historically catalysis has evolved as a set of different fields linked together by a unifying concept. While the \\ distinctions between the various areas serve a purpose, exciting work is happening at the interfaces.
}

C atalysis as a field has grown to encompass what had seemed to be disparate phenomena. The fact that fermentation of fruit to alcohol had a similar theoretical underpinning to the burning of coal gas on platinum wire would certainly not have been clear to the casual - or even determined - observer. It took the work of scientists such as Jacob Berzelius to generalize these phenomena as operating via a 'catalytic force', and the field of catalysis was (formally) born.

Still, catalysis remains a field more or less divided into the three classical disciplines: homogeneous, heterogeneous and biocatalysis. Although they have continually interacted, and ideas have passed from one to the other, divergences have also appeared. One well-known example is the difference in use of the term turnover number between chemo- and biocatalysis, related to the use of Michaelis-Menten kinetics almost exclusively for enzymatic reactions.

Of course these differences - in addition to differences in techniques and analysis - were not purely historical. Often there were very sound reasons why the fields used different approaches, which stem from the obvious differences that one encounters, for example, between working with syngas at several tens of bars versus using aqueous solutions of lipases for applications in detergents. And even from a procedural point of view, the legal and ethical issues relating to work with organisms are greater than those required for solid catalysts. But although it is certain that these differences can, and should, remain in the future, signs of convergence are also appearing thanks to greater understanding of catalytic systems.

For instance, insights into the active sites of enzymes arising from early protein crystallography helped to show the similarity between biocatalysis and homogeneous catalysis, and were a driver for the field of artificial enzyme research (as an aside, it is interesting to note that Berzelius vigorously opposed the idea that organisms were responsible for what we now know to be biocatalytic fermentation, believing instead that yeast was a simple contact catalyst behaving no differently to a metallic surface $\left.^{1}\right)$. And in the other direction we see continued efforts in designing enzymes to carry out work that had been exclusively the domain of synthetic homogenous systems - for instance, the formation of carbonsilicon bonds through directed evolution of cytochrome $c$ (ref. ${ }^{2}$ ).

\section{Catalysis as a field has grown to encompass what had seemed to be disparate phe- nomena. The fact that fermen- tation of fruit to alcohol had a similar theoretical underpin- ning to the burning of coal gas on platinum wire would certainly not have been clear to the casual - or even deter- mined - observer.}

Similarly, the relatively recent success achieved on single-atom catalysts has brought together researchers in homogeneous and heterogeneous fields, employing concepts and techniques from both areas. More generally, surface organometallic chemistry has a long history in bridging these disciplines, including at industrial scale ${ }^{3}$. Combining the ease of separation of heterogeneous systems with the potential for structure-activity relations of molecular catalysts is inherently appealing. But it is worth reminding ourselves that linking the heterogeneous and homogeneous worlds is not a panacea, and the inherent problems in one catalyst system are not necessarily solved by combining it with another. Despite the extensive work that has gone into the immobilization of molecular catalysts on solid supports, the resulting catalysts are yet to be successfully transferred to industry due to the increased costs involved, the currently observed reductions in performance, and the fact that immobilization of organometallic species does not necessarily solve the problem of catalyst degradation ${ }^{4}$. More work is needed to turn this dream into a (commercial) reality.

Some of these topics are discussed in far greater detail in the Perspective from Dean Toste, Gabor Somorjai and colleagues $^{5}$ in this issue. They take a look at combining fields at a fundamental level via the creation of hybrid catalysts. An initial examination of reactions that can be catalysed by all three classical catalyst classes (homogeneous, heterogeneous and enzymatic) leads onto a discussion of the methods for forming hybrids between these classes (heterogeneous-homogeneous, heterogeneous-enzyme and homogeneousenzyme), and the advantages and challenges of doing so.

Future developments in this area will require researchers who are fluent in the terminology and techniques of multiple sub-disciplines of catalysis, and who can identify those open problems where the fusion of different approaches can make the difference. Encouragingly, the academic research landscape has already given important signals in this direction, considering the growing number of established centres around the world focusing on multi-disciplinary catalysis already too numerous to mention here. And combined approaches have already shown their usefulness; for instance, commercial applications of glucose isomerase exclusively use the immobilized form due to the enhanced stability and recyclability afforded by the heterogeneity ${ }^{6}$.

The organically driven overlaps that continue to emerge are proving to be exciting and might lead to a more sustainable and efficient chemical industry. In any case, combining the strengths of each field - such as recyclability, tunability and specificity - while minimizing their corresponding weaknesses is a noble goal.

Published online: 11 May 2018

https://doi.org/10.1038/s41929-018-0088-3

References

1. Barnett, J. A. Microbiology 149, 557-567 (2003).

2. Kan, S. B. J., Lewis, R. D., Chen, K. \& Arnold, F. H. Science 354, 1048-1051 (2016)

3. Copéret, C. et al. Angew. Chem. Int. Ed. https://doi.org/10.1002/ anie.201702387 (2018).

4. Hübner, S., de Vries, J. G. \& Farina, V. Adv. Synth. Catal. 358, 3-25 (2016).

5. Ye, R., Zhao, J., Wickemeyer, B. B., Toste, F. D. \& Somorjai, G. A. Nat. Catal. https://doi.org/10.1038/s41929-018-0052-2 (2018).

6. Sheldon, R. A. \& Pereira, P. C. Chem. Soc. Rev. 46, 2678-2691. 